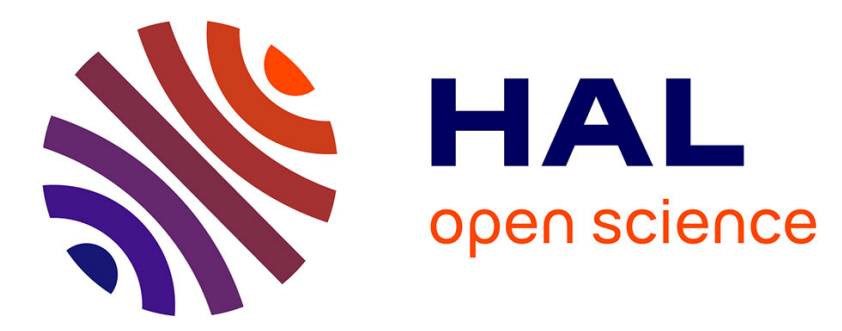

\title{
A note on non asymptotic stabilization of linear time delay systems
}

Wilfrid Perruquetti, Nicolás Espitia, Michel Dambrine

\section{To cite this version:}

Wilfrid Perruquetti, Nicolás Espitia, Michel Dambrine. A note on non asymptotic stabilization of linear time delay systems. 59th IEEE Conference on Decision and Control (CDC). December 14-18, 2020., Dec 2020, Jeju Island, South Korea. hal-03021373

\section{HAL Id: hal-03021373 https://hal.science/hal-03021373}

Submitted on 24 Nov 2020

HAL is a multi-disciplinary open access archive for the deposit and dissemination of scientific research documents, whether they are published or not. The documents may come from teaching and research institutions in France or abroad, or from public or private research centers.
L'archive ouverte pluridisciplinaire HAL, est destinée au dépôt et à la diffusion de documents scientifiques de niveau recherche, publiés ou non, émanant des établissements d'enseignement et de recherche français ou étrangers, des laboratoires publics ou privés. 


\section{A note on non asymptotic stabilization of linear time delay systems}

\author{
Wilfrid Perruquetti
}

\author{
Nicolás Espitia
}

\author{
Michel Dambrine
}

\begin{abstract}
This paper deals with fixed-time and prescribedtime stabilization of controllable linear-time delay systems using Artstein's transformation [1]. First, a delayed chain of integrators is stabilized in fixed-time using the desingularization technique originally introduced in [4]. Then, prescribed-time stabilization of controllable linear systems with input delay is obtained through Artstein's transformation combined with a new prescribed-time stabilizing control design (involving timevarying gains) for LTI systems. Compared to the control design methodology employed in e.g. [10], this new design offers an alternative somehow clearer way for the choice of the timevarying gains. Simulations illustrate the obtained results.
\end{abstract}

\section{INTRODUCTION}

Control design should successfully meet performances requested by end-users: reasonable energy consumption, quality of service, reliability.... Thus, in addition to stability \& robustness, another important criteria is the time for trajectories to reach a desired invariant mode (usually an equilibrium). Most of stabilization and estimation algorithms for practical engineering problems provide asymptotic or exponential convergence. However, when transient process has to be achieved in a given time (multi-agent rendezvous, missile guidance, ...), then non-asymptotic convergence becomes a central issue and a necessity.

Non-asymptotic convergences can be classified as follow: finite-time if the equilibrium is reached in a finite time dependent on initial conditions [21], [9], [2], [17]. If such a time is independent on initial deviations, then this type of convergence is called fixed-time [19]. If, additionally, for all initial conditions the time of convergence is constant, then such a property is called prescribed-time convergence [22], [25], [23], [24], [10].

Non-asymptotic (finite-time / fixed-time / prescribed-time) concepts have been extensively considered in the framework of linear and nonlinear ordinary differential equations (ODES) ([21], [9], [2], [17], [11], [18], [19], [20], [7], [15], [12]).

Finite-time / Fixed time stabilization of time delay systems are receiving more and more attention (see for example [13], [3]) since one of the pioneering papers, [16], which pointed out some key obstructions for the design of static finite-time controllers (see also [6]) and which came up with a controller based on Artstein's transformation to stabilize in finite-time with settling time depending on the initial conditions.

However, fixed-time \& prescribed time stabilization for linear time-delays systems (in particular those with input delay) have not been extensively investigated in the literature.

*N. Espitia and W. Perruquetti are with CRIStAL UMR 9189 CNRS \& M. Dambrine is with LAMIH UMR 8201 CNRS. Corresponding author: wilfrid.perruquetti@centralelille.fr
This paper provides new results on fixed-time \& prescribed time stabilization of some linear time-delay systems. We build on the Artstein' transformation [1] which allows to deal with a simpler system and from which we can perform an easier analysis for the control design for fixed-time or prescribed-time stabilization. For the fixed-time case, we bring back the ideas of [4] which exploits a "desingularization" technique, very helpful for the design of a control stabilizing a delayed chain of integrators in fixed time. Indeed, the proposed controller has terms with power less than one which imply non differentiability at zero of these terms, thus desingularization is used to circumvent this problem. For the prescribed-time case, we build on [22] and [10] where time-varying gains are used to achieve prescribedtime, independent of initial condition. Our approach makes uses of a Generalized polynomial-based Vandermonde matrix and the Exponential Bell polynomials. The paper is organized as follows: Section II provides the problem statement in III-A and some background (Artstein's transformation II-B non asymptotic stability II-C, II-D and III-E). Section III is devoted to the new obtained results: III-A provides a new fixed-time stabilizing controller for a delayed chain of integrators whereas III-B provides a new prescribed-time stabilizing controller for controllable linear systems with input delay. These results are supported by some simulation examples reported in section IV Then conclusion follows in $\mathrm{V}$

Notations: $\mathbb{R}_{+}$denotes the set of nonnegative real numbers. $\Gamma$ denotes the Gamma function. We define the signed power $a$ of a real number $x$ by $\{x\}^{a}=\operatorname{sign}(x)|x|^{a}$. Clearly, for $a \geq 1$, we have $\frac{d\{x\}^{a}}{d x}=a|x|^{a-1}$ and $\frac{d|x|^{a}}{d x}=$ $a\{x\}^{a-1}$. For non zero integers $m$ and $n$, let $0_{m \times n}$ be the $(m, n)$-matrix with zero entries, $I_{m}$ be the identity matrix of dimension $m, J_{n}=\left(\left(0_{(n-1) \times 1}, I_{n-1}\right)^{T}, 0_{n \times 1}\right)^{T}$ (Jordan matrix) and, for $p \in \mathbb{R}^{n}, L_{n}(p)=\left(0_{(n) \times(n-1)}, p\right)^{T}$. $L_{\text {loc }}^{1}(X)$ is the set of functions $f$ that are locally Lebesgue integrable over $X . \mathcal{C}^{k}(X, Y)$ is the set of functions $f: X \rightarrow$ $Y$ which are $k$ times continuously differentiable (noted as $\mathcal{C}^{k}$ when the sets $X, Y$ are obvious from the context). If $f \in \mathcal{C}^{k}$ then $f^{(k)}$ denotes its $k$-th derivative. $\mathcal{C} \mathcal{L}(X, Y)$ is the set of continuous (including at 0 ) functions $f: X \rightarrow Y$ which are locally Lipschitz everywhere except at 0 . A continuous function $\alpha:\left[0, a\left[\subset \mathbb{R}_{+} \rightarrow \mathbb{R}_{+}, r \mapsto \alpha(r)\right.\right.$, is said to be a class- $\mathcal{K}$ function if it is strictly increasing with $\alpha(0)=0$. $\alpha$ is a class- $\mathcal{K}_{\infty}$ function if it is a class- $\mathcal{K}$ function with $a=\infty$ and $\alpha(r) \rightarrow \infty$ as $r \rightarrow \infty$. A continuous function $\beta:\left[0,+\infty\left[\subset \mathbb{R}_{+} \times \mathbb{R} \rightarrow \mathbb{R}_{+},(r, t) \mapsto \beta(r, t)\right.\right.$, belongs to class- $\mathcal{K} \mathcal{L}$ if for each fixed $t$, the mapping $r \mapsto \beta(r, t)$ belongs 
to class $\mathcal{K}_{\infty}$ with respect to $r$. And, for each fixed $r \in \mathbb{R}_{+}$, the mapping $t \mapsto \beta(r, t)$ is decreasing with respect to $t$ and $\lim _{t \rightarrow+\infty} \beta(r, t)=0$.

\section{Problem Statement, CONCEPTS AND PRELIMINARIES}

\section{A. Problem statement}

Let us consider the following time-delay system:

$$
\dot{x}=A x+\sum_{i=1}^{k} B_{i} u\left(t-h_{i}\right), x \in \mathbb{R}^{n}, u \in \mathbb{R}, k \in \mathbb{N},
$$

where the delays $h_{i}$ are ordered $\left(0 \leq h_{1} \leq \ldots \leq h_{k}\right)$. The matrices $A, B_{i}$ are of appropriate dimensions and such that the pair $\left(A, B=\sum e^{h_{i}} B_{i}\right)$ satisfies the Kalman rank controllability condition for LTI systems. Thus $(A, B)$ can be assumed to be in the form $A=J_{n}+L_{n}(p)$ and $B=(0, \ldots, 0,1)^{T}$ without any loss of generality. We are interested in some non asymptotic stabilities of the origin for system (1): (uniform) "finite-time / fixed-time / prescribedtime stability " (respectively denoted by UFTS / UFxTS / UPrTS) and defined in subsection II-D.

For that, we will first transform the original TDS (1) into a non delayed one using some transformations (see subsection II-B]. The target closed-loop system will be of the form:

$$
\dot{z}=A z(t)+B u(t)=f(t, z), \quad z \in \mathbb{R}^{n}, u \in \mathbb{R} .
$$

Solutions of (2) with $z_{0}=z\left(t_{0}\right)$ are denoted by $\Phi^{t}\left(t_{0}, z_{0}\right)$.

\section{B. Transformations}

One of the first reduction methods for time-delay systems has been proposed by Kwon and Pearson in [14] for a system of form (1) with a single input delay (i.e. $k=2$ with $h_{1}=0$ ). A generalization of this work has been proposed by Artstein in [1] for linear system with general input delay described by

$$
\dot{x}(t)=A(t) x(t)+\int_{0}^{h_{k}} d \beta(\theta) u(t-\theta),
$$

where $\beta$ is a matrix-valued measure. This result applies to system (1): let

$$
z(t)=x(t)+\sum_{i=1}^{k} \int_{t-h_{i}}^{t} e^{\left(t-s-h_{i}\right) A} B_{i} u(s) d s,
$$

then $z(t)$ is a solution of the LTI system

$$
\dot{z}(t)=A z(t)+\left(\sum_{i=1}^{k} e^{-h_{i} A} B_{i}\right) u(t) .
$$

Theorem 7 from [16] explains under which conditions a stabilizing (resp. finite-time stabilizing) controller for (5) of the form $u(t, z)=k(t) l(z)$, makes the origin of ( 6 ) also asymptotically stable / finite-time stable with the same feedback with $z(t)$ replaced using (4). The conditions were $k(t)$ bounded and $l$ continuous such that $|l(z)| \leq \alpha(\|z\|)$ for some $\alpha$ a class $\mathcal{K}$-function. Thus, conclusion remains valid for fixed-time stability property (and will be used later on in section III), but not for UPrTS (see proof of Theorem 5).

\section{Non asymptotic stabilities of TDS}

Finite-time stabilization of TDS is very challenging. Indeed in [16], it has been shown that a TDS in the form

$$
\dot{x}(t)=f\left(x(t), x\left(t-h_{1}\right), \ldots, x\left(t-h_{k}\right)\right), x \in \mathbb{R}
$$

cannot be finite-time stable (FTS) if $f\left(x_{0}, \ldots, x_{k}\right)=0 \Leftrightarrow$ $x_{0}=\ldots=x_{k}$ ( $f$ is a nonzero continuous function). This is the reason why control with distributed delay were investigated for FTS using Artstein's transformation for $x^{(n)}=u(t-h), x \in \mathbb{R}, u \in \mathbb{R}$ or

$$
\dot{x}=a x(t)+\sum_{i=0}^{k} b_{i} u\left(t-h_{i}\right), x \in \mathbb{R}, u \in \mathbb{R}
$$

in the same paper. For example, (7) is UFTS with control

$$
\left.u(t)=-\frac{\left(a z(t)+\{z\}^{\alpha}(t)\right)}{\sum_{i=0}^{k} b_{i} e^{-a h_{i}}}, \alpha \in\right] 0,1[
$$

where $z$ is given by Artstein's transformation (4) which here reduce to: $z(t)=x(t)+\sum_{i=0}^{k} b_{i} \int_{-h_{i}}^{0} e^{a\left(-h_{i}-s\right)} u(t+s) d s$.

To best of our knowledge, except [26], no results exist concerning the other non asymptotic stability properties (Fixed-time stability \& prescribed-time stability). [26] is dealing with Fixed-time stabilization of controllable linear systems with delayed input. The obtained results are based on Artstein's transformation and on control for LTI system borrowed from [2], [19]. The here proposed controller is new, do not have strong restriction on the signed power terms, with only three parameters to tune.

\section{Non asymptotic concepts}

Stability properties of the non retarded system (2) are given in terms of $\mathcal{K} \mathcal{L}$-functions as follows:

Definition 1: The equilibrium $z=0$ of system (2) is said to be

- Uniformly finite-time stable (in short UFTS) if there exist a class $\mathcal{K} \mathcal{L}$ function $\beta$ and a constant $c>0$, independent of $t_{0}$, such that $\forall t \geq t_{0}, \forall z_{0}:\left\|z_{0}\right\|<c$,

$$
\left\|\Phi^{t}\left(t_{0}, z_{0}\right)\right\| \leq \beta\left(\left\|z_{0}\right\|, t-t_{0}\right),
$$

with $\beta\left(\left\|z_{0}\right\|, t\right)=0, \forall t \geq T\left(z_{0}\right)$,

- Uniformly fixed-time stable (in short UFxTS) if it is finite-time stable with $\sup _{\left\|z_{0}\right\|<c} T\left(z_{0}\right)<+\infty$.

- Uniformly stable in prescribed-time $T$ (in short UPrTS) if there exist a class $\mathcal{K} \mathcal{L}$ function $\beta$ and a positive constant $c$, independent of $t_{0}$, such that $\forall t>t_{0}$, $\forall z_{0}:\left\|z_{0}\right\|<c$,

$$
\left\|\Phi^{t}\left(t_{0}, z_{0}\right)\right\| \leq \beta\left(\left\|z_{0}\right\|, \mu_{T}\left(t-t_{0}\right)\right),
$$

where $\mu_{T}: \mathbb{R} \rightarrow \overline{\mathbb{R}}, \mu_{T}(t)=\frac{T}{T-t}$ for $t \in\left[t_{0}, t_{0}+\right.$ $T\left[\right.$ and $\mu_{T}(t)=+\infty, \forall t \in\left[t_{0}+T, \infty[\right.$. Note that $\lim _{t \rightarrow t_{0}+T} \mu_{T}\left(t-t_{0}\right)=+\infty$.

When $c=\infty$ in the previous relation then the corresponding notions are global.

Of course these definitions can be easily adapted for TDS (see for example [16], [6]). 


\section{E. Preliminary results}

Scalar case: In this subsection we review and complete some results about non-asymptotic stability for scalar systems of the form

$$
\dot{z}(t)=f(t, z(t)), \quad z \in \mathbb{R},
$$

where the function $f$ is assumed to be measurable and $L_{\text {loc }}^{1}(\mathbb{R} \times \mathbb{R})$ w.r.t $t$ and of $\mathcal{C} \mathcal{L}$-class w.r.t $z$. The solution of (9) with $z\left(t_{0}\right)=z_{0}$ is also denoted by $\Phi^{t}\left(t_{0}, z_{0}\right)$.

Finite-time stability: Let us consider (9) with $f(t, z)=$ $-r(z)$. The origin is assumed to be the unique equilibrium point, that is $r(z)=0 \Leftrightarrow z=0$. Solutions reach zero in a finite time $T=\int_{0}^{\left|z_{0}\right|} \frac{\mathrm{d} x}{r(x)}<\infty$ which leads to the following lemma ([9], [18]):

Lemma 1: 9pis globally UFTS iff $z r(z)>0, \forall z \in \mathbb{R} \backslash$ $\{0\}$ and $\int_{0}^{\left|z_{0}\right|} \frac{\mathrm{d} x}{r(x)}<\infty, \forall z_{0} \in \mathbb{R}$.

Fixed-time stability: Combining Lemma 1 with the second item of Definition [1 leads to:

Lemma 2: 9 is globally UFxTS iff $z r(z)>0, \forall z \in$ $\mathbb{R} \backslash\{0\}$ and $\sup _{z_{0} \in \mathbb{R}_{+}} \int_{0}^{z_{0}} \frac{\mathrm{d} x}{r(x)}<\infty$.

From which one can deduce the following result:

Theorem 1: For $0 \leq \alpha<1$ and $k>0, b>0$, system $\dot{z}=-k\{z\}^{\alpha} \exp (b|z|), z \in \mathbb{R}$ is globally UFxTS with settling time bounded as follows $T\left(z_{0}\right) \leq T_{\max }=\frac{\Gamma(1-\alpha)}{k b^{(1-\alpha)}}$. Moreover, any positive scalar function $V(t)$ satisfying the following differential inequality $\dot{V} \leq-k V^{\alpha} \exp (b|V|)$, decreases to zero in a fixed time less than $T_{\max }$.

Prescribed-time stability: The next Lemma gives sufficient conditions for UPrTS of the following scalar system:

$$
\dot{z}(t)=-c(t) z(t)
$$

Lemma 3: Let $c \in L^{1}(\mathbb{R})$ with $c(t)>0$ (a.e on $\mathbb{R}$ ), then 10 is UPrTS with prescribed-time $T>0$ if $\lim _{t \rightarrow t_{0}+T} \bar{c}(t)=+\infty$, where $\bar{c}(t):=\int_{t_{0}}^{t} c(\tau) \mathrm{d} \tau$.

There exist many functions meeting the sufficient condition for UPrTS in Lemma 3. Similar to [22], [10], we will consider "blow-up" functions having the form, for all $t \in\left[t_{0}, T+t_{0}\right):$

$$
c(t)=\frac{\left(c_{0} T\right)^{\epsilon}}{\left(T+t_{0}-t\right)^{\epsilon}}, \quad \epsilon>1, \quad c\left(t_{0}\right)=c_{0}^{\epsilon},
$$

so that 10 is UPrTS with prescribed-time $T+t_{0}$.

Time-varying linear systems in companion canonical form: Let us consider the case $f(t, z)=C(t) z$ with a companion matrix $C(t)=J_{n}+L_{n}(-p(t))$ and $p(t)$ to be characterized later on. The following theorem will be instrumental for the analysis of the UPrTS in the $n$-dimensional case and as we will apply in Subsection III-B

Theorem 2: Let us consider the nonlinear operators $\delta^{j}$ defined recursively by: $\left(\delta^{0} \rho\right)=\rho$ and $\left(\delta^{j+1} \rho\right)=\left(\rho+\frac{d}{d t}\right)\left(\delta^{j} \rho\right)$. Given $n$ distinct positive real numbers $r_{i}$ and a function $c(t)$ of the form (11), define the Generalized Vandermonde matrix

$$
V(t)=\left(\begin{array}{ccc}
1 & \cdots & 1 \\
\rho_{1}(t) & \cdots & \rho_{n}(t) \\
\left(\delta \rho_{1}\right)(t) & \cdots & \left(\delta \rho_{n}\right)(t) \\
\cdots & \cdots & \cdots \\
\left(\delta^{n-2} \rho_{1}\right)(t) & \cdots & \left(\delta^{n-2} \rho_{1}\right)(t)
\end{array}\right)
$$

and the companion matrix $C(t)=J_{n}+L_{n}(-p(t))$, with

$$
p(t)=V^{-\top}(t)\left(\begin{array}{c}
-\left(\delta^{n-1} \rho_{1}\right)(t) \\
\vdots \\
-\left(\delta^{n-1} \rho_{n}\right)(t)
\end{array}\right) \text {, }
$$

If $\rho_{i}(t)=-r_{i} c(t), i=1, \ldots, n$, yielding

$$
\begin{aligned}
\left(\delta^{j} \rho_{i}(t)\right) & =\sum_{k=0}^{j}\left(\begin{array}{l}
j \\
k
\end{array}\right) \rho_{i}^{(j-k)}(t) B_{k}\left(\rho_{i}(t), \dot{\rho}_{i}(t), \ldots, \rho_{i}^{(k-1)}(t)\right) \\
& =B_{j+1}\left(\rho_{i}(t), \dot{\rho}_{i}(t), \ldots, \rho_{i}^{(j)}(t)\right) .
\end{aligned}
$$

$j=0, . ., n-2$, where $B_{k}(\cdot)$ denotes the complete Bell polynomials.

Then, solutions of (2) with $f(t, z)=C(t) z$ satisfy:

$$
\|z(t)\| \leq P(\sqrt{c(t)}) \exp \left(-r_{\min } \bar{c}(t)\right)\left\|z_{0}\right\|,
$$

where $r_{\min }=\min _{i} r_{i}>0, P$ is a polynomial of degree at most $n-1$ and $\bar{c}(t)=\int_{t_{0}}^{t} c(s) \mathrm{d} s$. Moreover (2) is UPrTS with $T>0$.

Proof: The proof is given in [8], where the domination of the polynomial by the "exp" function is used.

\section{MAIN RESULTS}

\section{A. Fixed-time stabilization of a delayed chain of integrators}

Let us consider the following delayed chain of integrators:

$$
\dot{x}_{1}=x_{2}\left(t-h_{1}\right), \ldots, \dot{x}_{n}=v\left(t-h_{n}\right),
$$

where the state ${ }^{1}$ is $x_{t} \in \mathcal{C}^{0}\left(\mathbb{R}^{n} \times \mathbb{R}^{n}\right)$, the control is $v \in L^{1}(\mathbb{R})$ and $h_{i}, i=1, \ldots, n$ are known constant delays. Setting $\tilde{h}_{i}=\sum_{j=0}^{i-1} h_{j}, h_{0}=0$, the change of variable $\tilde{x}_{i}(t)=x_{i}\left(t-\tilde{h}_{i}\right)$, for $i=1, \ldots, n$, leads to

$$
\frac{d \tilde{x}(t)}{d t}=J_{n} \tilde{x}(t)+B v\left(t-\tilde{h}_{n+1}\right)
$$

where $B=(0, \ldots, 0,1)^{\top}$. Thus, Artstein's transformation (4) applied to system (17) is given by:

$$
z(t)=\exp \left(\tilde{h}_{n+1} J_{n}\right) \tilde{x}(t)+\int_{-\tilde{h}_{n+1}}^{0} \exp \left(-s J_{n}\right) B v(t+s) d s,
$$

which, using coordinates $x=\left(x_{1}, \ldots, x_{n}\right)$, reads as:

$$
\begin{aligned}
z_{1}(t) & =\sum_{k=1}^{n} \frac{\tilde{h}_{n+1}^{k-1}}{(k-1) !} x_{k}\left(t-\tilde{h}_{k}\right)+\int_{-\tilde{h}_{n+1}}^{0} \frac{(-s)^{n-1}}{(n-1) !} v(t+s) d s \\
z_{i}(t) & =\sum_{k=i}^{n} \frac{\tilde{h}_{n+1}^{k-i}}{(k-i) !} x_{k}\left(t-\tilde{h}_{k}\right)+\int_{-\tilde{h}_{n+1}}^{0} \frac{(-s)^{n-i}}{(n-i) !} v(t+s) d s \\
z_{n}(t) & =x_{n}\left(t-\tilde{h}_{n+1}\right)+\int_{-\tilde{h}_{n+1}}^{0} v(t+s) d s
\end{aligned}
$$

${ }^{1} x_{t}$ is defined by $x_{t}(s)=x(t+s)$ with $s \in\left[-\max _{i} h_{i}, 0\right]$. 
Now, using this transformation, system becomes $\dot{z}(t)=$ $J_{n} z(t)+B v(t)$ or equivalently

$$
\dot{z}_{1}(t)=z_{2}(t), \ldots, \dot{z}_{n}(t)=v(t) .
$$

For any vector $z \in \mathbb{R}^{n}$ we use the following notation: $\bar{z}_{i}=$ $\left(z_{1}, \ldots, z_{i}\right)$. Following [11] and [5], let us set $z_{1}^{\star}=0, \zeta_{1}=$ $z_{1}$ and then define recursively for $i=1, \ldots, n-1$ :

$$
\begin{aligned}
z_{i+1}^{\star} & =-\left\{\zeta_{i}\right\}^{\alpha_{i}} \gamma_{i}\left(\bar{z}_{i}\right), \\
\zeta_{i+1} & =\left\{z_{i+1}\right\}^{\frac{1}{\alpha_{i}}}-\left\{z_{i+1}^{\star}\right\}^{\frac{1}{\alpha_{i}}},
\end{aligned}
$$

where $\alpha_{i}$ are defined by $\alpha_{0}=1$ and for $i \geq 1$ :

$$
\alpha_{i}=\alpha_{i-1}+2(\alpha-1) .
$$

We have $\alpha_{i}=1-2 i(1-\alpha), \forall i=0, \ldots, n$. Let us select $\alpha$ such that $1-\frac{1}{2 n}<\alpha<1$, so that $1-i / n<\alpha_{i}<1$ and $0<\alpha_{n}<\ldots<\alpha_{1}<1$. For $i=1, \ldots, n$, let us define:

$$
\begin{aligned}
\gamma_{i}\left(\bar{z}_{i}\right) & =\frac{2}{\alpha}+(n-i)+k n^{\alpha-1} 2^{\alpha} e^{2 n b \zeta_{i}^{2}}+\eta_{i}\left(\bar{z}_{i-1}\right), \\
\eta_{i}\left(\bar{z}_{i-1}\right) & =(i-1) \frac{(2 \alpha-1)^{2 \alpha-1}}{\alpha^{2 \alpha}}\left(d_{i}\left(\bar{z}_{i-1}\right)\right)^{2 \alpha}, \\
Q_{i}\left(\bar{z}_{i}\right) & =\int_{z_{i}^{\star}}^{z_{i}}\left\{\{s\}^{\frac{1}{\alpha_{i-1}}}-\left\{z_{i}^{\star}\right\}^{\frac{1}{\alpha_{i-1}}}\right\}^{2-\alpha_{i-1}} d s, \\
V_{i}\left(\bar{z}_{i}\right) & =V_{i-1}\left(\bar{z}_{i-1}\right)+Q_{i}\left(\bar{z}_{i}\right)=\sum_{j=1}^{i} Q_{j}\left(\bar{z}_{j}\right), \\
W_{i}\left(\bar{z}_{i}\right) & =k n^{\alpha-1}\left(2 \zeta_{i}^{2}\right)^{\alpha} e^{2 n b \zeta_{i}^{2}}
\end{aligned}
$$

where $V_{0}\left(\bar{z}_{0}\right)=0$ and $d_{i}\left(\bar{z}_{i-1}\right) \in \mathcal{C}^{1}$ is given in Lemma 9 (see Appendix). Thus from (24), we have $\eta_{i}\left(\bar{z}_{i-1}\right) \in \mathcal{C}^{1}$ which imply that $\gamma_{i}\left(\bar{z}_{i}\right)$ and $\left\{z_{i}^{\star}\right\}^{\frac{1}{\alpha_{i-1}}}$ are also $\mathcal{C}^{1}$.

Proposition 1: For $i=1, \ldots, n$ the following hold:

(L1) $0 \leq V_{i}\left(\bar{z}_{i}\right) \leq 2 \sum_{j=1}^{i} \zeta_{j}^{2}$,

(L2) $\dot{V}_{i}\left(\bar{z}_{i}\right) \leq-\sum_{j=1}^{i} W_{j}\left(\bar{z}_{j}\right)-(n-i) \sum_{j=1}^{i}\left|\zeta_{j}\right|^{2 \alpha}+$ $\frac{\alpha_{i}}{\alpha}\left|\zeta_{i+1}\right|^{2 \alpha}$.

Note that $V_{i}\left(\bar{z}_{i}\right) \geq 0$ means that $V_{i}\left(\bar{z}_{i}\right)$ is positive definite with respect to $\bar{z}_{i}$.

Proof: The proof is done by induction.

Step $i=1$ : We have $V_{1}\left(z_{1}\right)=Q_{1}\left(z_{1}\right)=\int_{0}^{z_{1}} s \mathrm{~d} s=\frac{1}{2} z_{1}^{2} \leq$

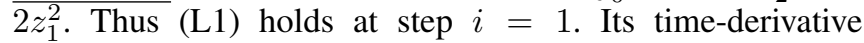
satisfies:

$$
\begin{aligned}
\dot{V}_{1} & =z_{1} z_{2}^{\star}+z_{1}\left(z_{2}-z_{2}^{\star}\right) \\
& \leq z_{1} z_{2}^{\star}+2\left|z_{1}\right|\left|\zeta_{2}\right|^{\alpha_{1}} \\
& \leq z_{1} z_{2}^{\star}+\frac{1}{\alpha}\left|z_{1}\right|^{2 \alpha}+\frac{\alpha_{1}}{\alpha}\left|\zeta_{2}\right|^{2 \alpha}
\end{aligned}
$$

The first inequality comes from 41, the second from (44) using $\alpha_{1}+1=2 \alpha$. From (20) and 23), as $\eta_{1}=0$, we get

$$
z_{1} z_{2}^{\star}=-W_{1}\left(z_{1}\right)-\left|z_{1}\right|^{2 \alpha}\left(\frac{2}{\alpha}+(n-1)\right) .
$$

Replacing in the previous inequality, we obtain

$$
\dot{V}_{1}\left(z_{1}\right) \leq-W_{1}\left(z_{1}\right)-(n-1)\left|z_{1}\right|^{2 \alpha}+\frac{\alpha_{1}}{\alpha}\left|\zeta_{2}\right|^{2 \alpha},
$$

which means that (L2) holds at step $i=1$.

Step $i$ : Assume that (L1), (L2) hold at step $i-1$. Since $\overline{\gamma_{i-1} \in \mathcal{C}^{1}}$ we deduce that $z_{i}^{\star} \in \mathcal{C}^{1}\left(\mathbb{R}^{i} \backslash\{0\}\right)$ but $\left\{z_{i}^{\star}\right\}^{\frac{1}{\alpha_{i-1}}} \in$ $\mathcal{C}^{1}$. Thus $Q_{i}\left(\bar{z}_{i}\right)$ given by 25] is clearly $\mathcal{C}^{1}$ because $(2-$ $\left.\alpha_{i-1}\right)>1$. As $z \rightarrow\{z\}^{\mu}$ is an increasing function for $\mu \geq$
0 , then $Q_{i}\left(\bar{z}_{i}\right) \geq 0$, and $Q_{i}\left(\bar{z}_{i}\right)=0 \Leftrightarrow z_{i}^{\star}=z_{i}$. This involves at its turn that $V_{i}$ is a positive definite function. Using 422 (see Appendix) we get $V_{i}\left(\bar{z}_{i}\right) \leq V_{i-1}\left(\bar{z}_{i-1}\right)+$ $2 \zeta_{i}^{2} \leq 2 \sum_{j=1}^{i} \zeta_{j}^{2}$. Thus (L1) holds also at step $i$.

Since $Q_{i}$ is differentiable, then we have $\dot{Q}_{i}=$ $\sum_{j=1}^{i-1} \frac{\partial Q_{i}}{\partial z_{j}} z_{j+1}+\left\{\zeta_{i}\right\}^{2-\alpha_{i-1}} z_{i+1}$, where we have used the fact that $\frac{\partial Q_{i}}{\partial z_{i}}=\left\{\zeta_{i}\right\}^{2-\alpha_{i-1}}$. Now, $\forall j: 1 \leq j<i$ we have:

$$
\begin{aligned}
\frac{\partial Q_{i}}{\partial z_{j}} & =\frac{\partial Q_{i}}{\partial z_{i}^{\star}} \frac{\partial z_{i}^{\star}}{\partial z_{j}}, \\
\frac{\partial Q_{i}}{\partial z_{j}} & =-G_{i, j}\left(\bar{z}_{i-1}\right) \int_{z_{i}^{\star}}^{z_{i}}\left|\{s\}^{\frac{1}{\alpha_{i-1}}}-\left\{z_{i}^{\star}\right\}^{\frac{1}{\alpha_{i-1}}}\right|^{1-\alpha_{i-1}} d s, \\
G_{i, j}\left(\bar{z}_{i-1}\right) & =\frac{2-\alpha_{i-1}}{\alpha_{i-1}}\left|z_{i}^{\star}\right|^{\frac{1-\alpha_{i-1}}{\alpha_{i-1}}} \frac{\partial z_{i}^{\star}}{\partial z_{j}}, \\
\left|\frac{\partial Q_{i}}{\partial z_{j}}\right| & \leq 2 G_{i, j}\left(\bar{z}_{i-1}\right)\left|\zeta_{i}\right| .
\end{aligned}
$$

The last inequality is obtained using (42). Using (44) we get:

$$
\begin{aligned}
& 2\left|\zeta_{j}\right|^{2 \alpha-1}\left|\zeta_{i}\right| d_{i-1}\left(\bar{z}_{i-1}\right) \\
& \leq\left|\zeta_{j}\right|^{2 \alpha}+\frac{(2 \alpha-1)^{2 \alpha-1}}{\alpha^{2 \alpha}}\left(\left|\zeta_{i}\right| d_{i-1}\left(\bar{z}_{i-1}\right)\right)^{2 \alpha},
\end{aligned}
$$

which combined with 50 (Lemma 9 Appendix) gives:

$$
\begin{gathered}
2 \sum_{j=1}^{i-1} G_{i, j}\left(\bar{z}_{i-1}\right)\left|z_{j+1}\right|\left|\zeta_{i-1}\right|^{1-\alpha_{i-1}}\left|\zeta_{i}\right| \leq \\
\sum_{j=1}^{i-1}\left|\zeta_{j}\right|^{2 \alpha}+(i-1) \frac{(2 \alpha-1)^{2 \alpha-1}}{\alpha^{2 \alpha}}\left(\left|\zeta_{i}\right| d_{i}\left(\bar{z}_{i-1}\right)\right)^{2 \alpha} .
\end{gathered}
$$

Thus, from (L2) at step $i-1$ and 29], we have

$$
\begin{aligned}
\dot{V}_{i} \leq & -\sum_{j=1}^{i-1} W_{j}\left(\bar{z}_{j}\right)-(n-(i-1)) \sum_{j=1}^{i-1}\left|\zeta_{j}\right|^{2 \alpha}+\frac{\alpha_{i-1}}{\alpha}\left|\zeta_{i}\right|^{2 \alpha} \\
& +\sum_{j=1}^{i-1}\left|\zeta_{j}\right|^{2 \alpha}+(i-1) \frac{(2 \alpha-1)^{2 \alpha-1}}{\alpha^{2 \alpha}}\left(\left|\zeta_{i}\right| d_{i}\left(\bar{z}_{i-1}\right)\right)^{2 \alpha} \\
& +\left\{\zeta_{i}\right\}^{2-\alpha_{i-1}}\left(z_{i+1}^{\star}+\left(z_{i+1}-z_{i+1}^{\star}\right)\right) .
\end{aligned}
$$

Using (41] we have

$$
\left|\left\{\zeta_{i}\right\}^{2-\alpha_{i-1}}\left(z_{i+1}-z_{i+1}^{\star}\right)\right| \leq 2\left|\zeta_{i}\right|^{2-\alpha_{i-1}}\left|\zeta_{i+1}\right|^{\alpha_{i}},
$$

which combined with 44 leads to

$$
\left|\left\{\zeta_{i}\right\}^{2-\alpha_{i-1}}\left(z_{i+1}-z_{i+1}^{\star}\right)\right| \leq \frac{2-\alpha_{i-1}}{\alpha}\left|\zeta_{i}\right|^{2 \alpha}+\frac{\alpha_{i}}{\alpha}\left|\zeta_{i+1}\right|^{2 \alpha} .
$$

Replacing $z_{i+1}^{\star}$ by $-\left\{\zeta_{i}\right\}^{\alpha_{i}} \gamma_{i}\left(\bar{x}_{i}\right)$ in light of (20) (where $\gamma_{i}$ is given by (23) ) and using $2+\alpha_{i}-\alpha_{i-1}=2 \alpha$, we get:

$$
\begin{aligned}
\dot{V}_{i} \leq & -\sum_{j=1}^{i-1} W_{j}\left(\bar{z}_{j}\right)-W_{i}\left(\bar{z}_{i}\right)-(n-i) \sum_{j=1}^{i-1}\left|\zeta_{j}\right|^{2 \alpha} \\
& -(n-i)\left|\zeta_{i}\right|^{2 \alpha}+\frac{\alpha_{i}}{\alpha}\left|\zeta_{i+1}\right|^{2 \alpha}
\end{aligned}
$$

which is (L2) at step $i$. This concludes the proof.

Now, we are ready to state our main result about FxTS of a delayed chain of integrators:

Theorem 3: System 16 is globally UFxTS under the following feedback control:

$$
v(t)=-\left\{\zeta_{n}(t)\right\}^{1+2 n(\alpha-1)} \gamma_{n}(t),
$$


where $\zeta_{n}(t), \gamma_{n}(t)$ are respectively given by 21, , 23, together with $z_{i}, z_{i}^{\star}, \alpha_{i}, \delta_{i}$ respectively given by (18), (20), [22], 24) and with parameters:

- $1-\frac{1}{2 n}<\alpha<1$,

- $k$ and $b$ are positive free parameters to be tuned for selecting the settling-time bound $T_{\max }=\frac{\Gamma(1-\alpha)}{k b^{(1-\alpha)}}+$ $\sum_{j=1}^{n} h_{j}$.

Proof: It follows by Proposition 1 combined with (46) and Theorem 7 from [16].

\section{B. Prescribed-time stabilization}

In this subsection, we apply Theorem 2 with $c$ defined by (11), to get UPrTS of a chain of integrators.

Theorem 4: The system

$$
\frac{d \bar{z}}{d t}(t)=A \bar{z}(t)+B u(t), \quad \bar{z} \in \mathbb{R}^{n}, u \in \mathbb{R},
$$

with $A=J_{n}, B=(0, \ldots, 0,1)^{\top}$, is UPrTS under the control

$$
u(t)=-\sum_{i=1}^{n} p_{i}(t) \bar{z}_{i}(t),
$$

with $p(t)=\left(p_{1}(t), \ldots, p_{n}(t)\right)^{\top}$ given by (13) with $V(t)$ given by (12) in which the operator $\left(\delta^{n} \rho(t)\right)$ is defined recursively by: $\left(\delta^{0} \rho\right)=\rho,\left(\delta^{i+1} \rho\right)=\left(\rho+\frac{d}{d t}\right)\left(\delta^{i} \rho\right)$ (characterized by (14)), the function $c$ is defined by (11) and the coefficients $r_{i}, i=1, \ldots, n$ are different positive real numbers $\left(r_{i}>0, r_{i} \neq r_{j}\right.$ for $i \neq j$ in the range $\left.1, \ldots n\right)$.

If we apply Artstein's transformation (4) to the linear timedelay system (1), then we obtain the LTI system (5) which is in the form $\dot{z}=A z(t)+B u(t)$ with $B=\sum e^{h_{i}} B_{i}$. Assuming that $\left(A, B=\sum e^{h_{i}} B_{i}\right)$ is controllable, there exists a linear transformation $\mathbb{T}$ such that setting $\bar{z}=\mathbb{T}^{-1} z$ the obtained differential equation reads as (33) for which Theorem 4 applies. Let us recall that the matrix $\mathbb{T}$ is given by:

$$
\mathbb{T}=\left(A^{n-1}, \ldots, I_{n}\right) B, \quad B=\sum e^{h_{i}} B_{i} .
$$

Thus, we have the following result:

Theorem 5: Let us assume that $\left(A, B=\sum e^{h_{i}} B_{i}\right)$ is controllable and let $\mathbb{T}$ be given by (35). Let us define the control $u(t)$ by (34) where $\bar{z}=\mathbb{T}^{-1} z$ and $z$ is given by (4), $p(t)=\left(p_{1}(t), \ldots, p_{n}(t)\right)^{\top}$ given by (13) in which the operator $\delta^{n} \rho(t)$ is defined recursively by: $\delta^{0} \rho=\rho, \delta^{i+1} \rho=$ $\left(\rho+\frac{d}{d t}\right) \delta^{i} \rho$, the function $c$ is defined by 111$)$ and the coefficients $r_{i}, i=1, \ldots, n$ are different positive real numbers $\left(r_{i}>0, r_{i} \neq r_{j}\right.$ for $i \neq j$ in the range $\left.1, \ldots n\right)$. Then, the linear time-delay system (11) with feedback (34) is globally UPrTS with prescribed-time $T+\max _{i} h_{i}>0$.

\section{Simulations}

\section{A. Unstable first-order system with input delay}

Let us consider the equation

$$
\dot{x}(t)=x(t)+u(t-h), \quad x(t) \in \mathbb{R},
$$

with $h=0.2 \mathrm{~s}$. Using Artstein's transformation $z(t)=$ $x(t)+\int_{-h}^{0} e^{(-h-s)} u(t+s) d s$, we get the reduced system
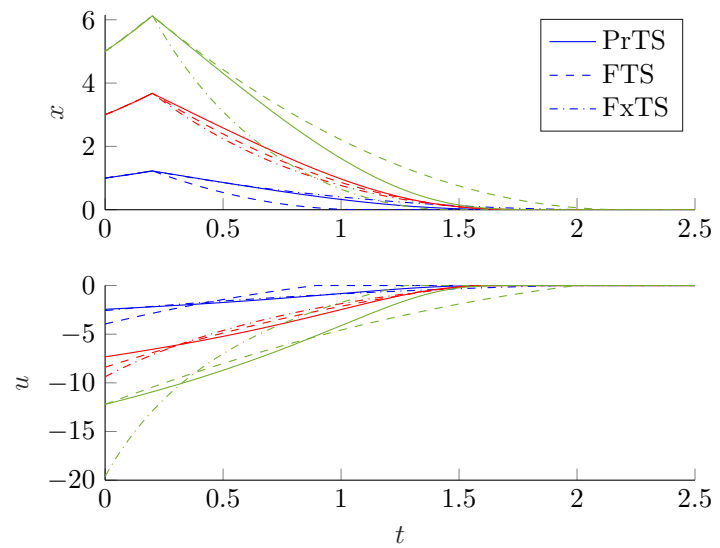

Fig. 1. Finite-time stabilization of system 36 for 3 different initial conditions; solid line: control law 39, dashed: control law 38, dotted: control law 37

$\dot{z}(t)=z(t)+e^{-h} u(t)$. Applying previous results, we get three control laws:

$$
u(t)=-e^{h}\left(z(t)+k_{1}\{z(t)\}^{0.5}\right),
$$

$($ FxTS $) \quad u(t)=-e^{h}\left(z(t)+k_{2}\{z(t)\}^{0.5}\right) e^{0.1 z(t)}$,

$(\operatorname{PrTS}) \quad u(t)=-e^{h}(1+c(t)) z(t)$,

with $k_{1}=\sqrt{5}, k_{2}=\frac{\sqrt{10 \pi}}{2}$ and if $t<2, c(t)=\left(1-\frac{t}{2}\right)^{-2}$, else $c(t)=100$. Fig. 1 shows the simulation results for three different initial conditions.

\section{B. Delayed double integrator}

We consider here the system

$$
\begin{aligned}
& \dot{x}_{1}=x_{2}\left(t-h_{1}\right), \\
& \dot{x}_{2}=u\left(t-h_{2}\right),
\end{aligned}
$$

with $h_{1}=0.2 \mathrm{~s}$ and $h_{2}=0.1 \mathrm{~s}$. Using the transformation defined in (18), this system is reduced to a standard double integrator system. Applying the control (34) with $p_{1}(t)=$ $r_{1} r_{2} c^{2}(t)$ and $p_{2}(t)=\left(r_{1}+r_{2}\right) c(t)-2 \frac{\sqrt{c(t)}}{c_{0} T}$ and $\epsilon=2$, $T=1, r_{1}=1, r_{2}=2$, we get for an initial condition $x_{1}(0)=-2$ and $x_{2}(\theta)=2, \forall \theta \in\left[-h_{1}, 0\right]$ the response plotted in red Fig. 2 .

We also apply control (32) with $\alpha=0.8, k=10^{-4}$, and $b=10^{-3}$. The response of the closed-loop system is given in Fig. 2 in blue.

\section{CONCLUSiON}

In this paper, we have addressed the problem of Fixedtime \& Prescribed-time stabilization of some classes of timedelay systems. By relying on Arstein's transformation and studying a delayed chain of integrators, we have designed new controllers to achieve fixed-time / prescribed-time stability. Future work includes extension to more general class of linear TDS and robustness properties with respect to some class of perturbations; in particular by exploiting our obtained fixed-time stabilizer controller and by virtue of its Lyapunov-based construction. For that we will start with a perturbed chain of integrators. 

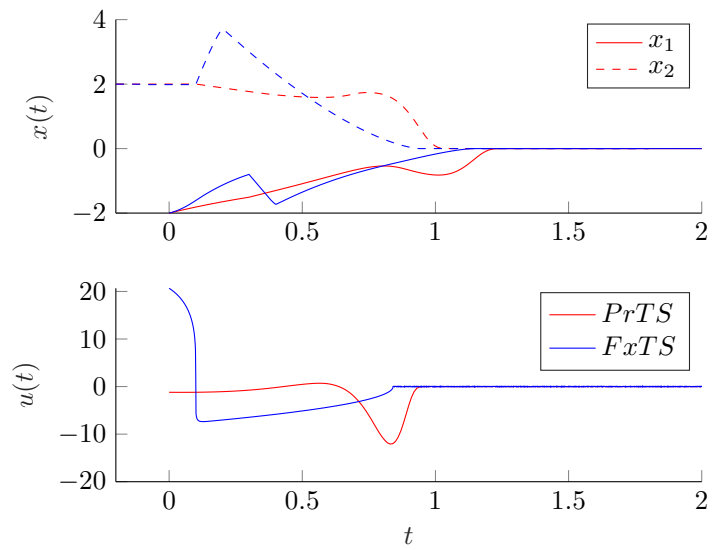

Fig. 2. Finite-time stabilization of system 40, using control 34 with control laws 34] (in red) and (32) (in blue).

\section{APPENDIX}

Lemma 4: $\forall(x, y) \in \mathbb{R}^{2}, \forall \mu>1, \forall \nu>0$ the following inequalities hold:

$$
\begin{array}{r}
|x-y| \leq 2\left|\{y\}^{\mu}-\{x\}^{\mu}\right|^{1 / \mu}, \\
0 \leq \int_{x}^{y}\left\{\{s\}^{\mu}-\{x\}^{\mu}\right\}^{\nu} d s \leq 2\left|\{y\}^{\mu}-\{x\}^{\mu}\right|^{\nu+\frac{1}{\mu}} .
\end{array}
$$

Lemma 5: $\forall(x, y) \in \mathbb{R}_{+}^{2}$ and $\forall(p, q) \in \mathbb{R}_{+}^{2}, \frac{1}{p}+\frac{1}{q}=1$, the following Young's inequality holds:

$$
x y \leq \frac{x^{p}}{p}+\frac{y^{q}}{q} .
$$

Using (43), setting $p=\frac{a+b}{a}, q=\frac{a+b}{b}$ and $x \rightarrow x^{a} z^{\frac{a c}{a+b}}, y \rightarrow$ $z^{-\frac{a c}{a+b}} y^{b}(\rightarrow$ means "is replaced by"), one has:

Lemma 6: $\forall(x, y) \in \mathbb{R}_{+}^{2}, z>0$ and $\forall(a, b, c) \in \mathbb{R}_{+}^{3}$, the following inequality holds:

$$
x^{a} y^{b} \leq \frac{a}{a+b} z^{c} x^{a+b}+\frac{b}{a+b} z^{-\frac{a c}{b}} y^{a+b} .
$$

Lemma 7: For a real convex function $f$, numbers $x_{1}, x_{2}, \ldots, x_{n}$ in its domain, Jensen's inequality can be stated as

$$
n f\left(\frac{1}{n} \sum_{i=1}^{n} x_{i}\right) \leq \sum_{i=1}^{n} f\left(x_{i}\right) .
$$

Lemma 8: For any $V_{i}>0,0<\alpha<1, b>0$ the following inequality holds $\left(V=\sum_{i=1}^{n} V_{i}\right)$ :

$$
-n^{\alpha-1} \sum_{i=1}^{n} V_{i}^{\alpha} \exp \left(2 b V_{i}\right) \leq-V^{\alpha} \exp (b V) .
$$

Lemma 9: $\forall i=1, \ldots, n$ and $\forall j: 1 \leq j<i$, the following inequalities hold:

$$
\begin{gathered}
\left|G_{i, j}\left(\bar{z}_{i-1}\right)\right| \leq\left(\sum_{\ell=1}^{i-1}\left|\zeta_{\ell}\right|^{1-\alpha_{j-1}}\right) a_{i, j}\left(\bar{z}_{i-1}\right), \\
\left|z_{j+1}\right| \leq\left(\sum_{\ell=1}^{j+1}\left|\zeta_{\ell}\right|^{\alpha_{j}}\right) b_{j}\left(\bar{z}_{j}\right), \\
\left|G_{i, j}\left(\bar{z}_{i-1}\right) z_{j+1}\right| \leq\left(\sum_{\ell=1}^{i-1}\left|\zeta_{\ell}\right|^{2 \alpha-1}\right) c_{j}\left(\bar{z}_{i-1}\right), \\
\left|\sum_{j=1}^{i-1} G_{i, j}\left(\bar{z}_{i-1}\right) z_{j+1}\right| \leq\left(\sum_{\ell=1}^{i-1}\left|\zeta_{\ell}\right|^{2 \alpha-1}\right) d_{i}\left(\bar{z}_{i-1}\right),
\end{gathered}
$$

where all functions $a_{i, j}, b_{j}, c_{j}, d_{i}$ are $\mathcal{C}^{1}$ functions.

\section{REFERENCES}

[1] Z. Artstein. Linear systems with delayed controls: A reduction. IEEE Trans. Autom. Control, 27(4):869-879, Aug 1982.

[2] S.P. Bhat and D.S. Bernstein. Finite-time stability of continuous autonomous systems. SIAM J. Control Optim., 38(3):751-766, 2000.

[3] G. Chen and Y. Yang. New sufficient conditions for finite time stability of nonlinear time delay systems. Asian Journal of Control, 21(5):2321-2329, 2019.

[4] J.-M. Coron and L. Praly. Adding an integrator for the stabilization problem. Systems \& Control Letters, 17(2):89 - 104, 1991.

[5] Brigitte d'Andréa Novel, Jean-Michel Coron, and Wilfrid Perruquetti. Small-time stabilization of nonholonomic or underactuated mechanical systems: the unicycle and the slider examples, 2019.

[6] D. Efimov, A. Polyakov, E. Fridman, W. Perruquetti, and J.-P. Richard. Comments on finite-time stability of time-delay systems. Automatica, 50(7): 1944 - 1947, 2014.

[7] D. Efimov, A. Polyakov, W. Perruquetti, and J. . Richard. Weighted homogeneity for time-delay systems: Finite-time and independent of delay stability. IEEE Trans. Autom. Control, 61(1):210-215, Jan 2016.

[8] N. Espitia and W. Perruquetti. Predictor-feedback prescribed-time stabilization of LTI systems with input delay. Under review in IEEE Transaction on Automatic Control, 2020.

[9] V. T. Haimo. Finite time controllers. SIAM J. Control Optim., 24(4):760-770, 1986

[10] J. Holloway and M. Krstic. Prescribed-time output feedback for linear systems in controllable canonical form. Automatica, 107:77-85, 2019.

[11] X. Huang, W. Lin, and B. Yang. Global finite-time stabilization of a class of uncertain nonlinear systems. Automatica, 41(5):881 - 888, 2005

[12] E. Jiménez-Rodríguez, AJ. Munoz-Vázquez, JD. Sánchez-Torres, M. Defoort, and AG. Loukianov. A Lyapunov-like characterization of predefined-time stability. IEEE Trans. Autom. Control, pages 1-1, 2020 (Early paper)

[13] I. Karafyllis. Finite-time global stabilization by means of time-varying distributed delay feedback. SIAM J. Control Optim., 45:320-342, 2006.

[14] W. Kwon and A. Pearson. Feedback stabilization of linear systems with delayed control. IEEE Trans. Autom. Control, 25(2):266-269, Apr 1980

[15] F. Lopez-Ramirez, A. Polyakov, D. Efimov, and W. Perruquetti. Finitetime and fixed-time observer design: Implicit Lyapunov function approach. Automatica, 87:52 - 60, 2018.

[16] E. Moulay, M. Dambrine, N. Yeganefar, and W. Perruquetti. Finitetime stability and stabilization of time-delay systems. Systems \& Control Letters, 57:561-566, 2008.

[17] E. Moulay and W. Perruquetti. Finite time stability of nonlinear systems. In 42nd IEEE International Conference on Decision and Control, volume 4, pages 3641-3646 vol.4, Dec 2003.

[18] E. Moulay and W. Perruquetti. Finite time stability and stabilization of a class of continuous systems. Journal of Mathematical Analysis and Applications, 323(2):1430 - 1443, 2006.

[19] A. Polyakov. Nonlinear feedback design for fixed-time stabilization of linear control systems. IEEE Trans. Autom. Control, 57(8):2106-2110, Aug 2012.

[20] A. Polyakov, D. Efimov, and W. Perruquetti. Finite-time and Fixedtime Stabilization: Implicit Lyapunov Function Approach. Automatica, 51(1):332-340, 2015.

[21] E Roxin. On finite stability in control systems. Rendiconti del Circolo Matematico di Palermo, 15:273-280, 1966

[22] Y.-D. Song, Y.-J. Wang, J.-C. Holloway, and M. Krstic. Timevarying feedback for regulation of normal-form nonlinear systems in prescribed finite time. Automatica, 83:243 - 251, 2017

[23] Y.-D. Song, Y.-J. Wang, and M. Krstic. Time-varying feedback for stabilization in prescribed finite time. International Journal of Robust and Nonlinear Control, 2018.

[24] D. Steeves, M. Krstic, and R. Vazquez. Prescribed-time $H^{1}$ stabilization of reaction-diffusion equations by means of output feedback. In 18th European Control Conference (ECC), pages 1932-1937, June 2019.

[25] Y. Wang, Y. Song, D. J. Hill, and M. Krstic. Prescribed-time consensus and containment control of networked multiagent systems. IEEE Trans. Cybern., 49(4):1138-1147, 2018.

[26] Z. Zuo. Fixed-time stabilization of general linear systems with input delay. Journal of the Franklin Institute, 356(8):4467 - 4477, 2019. 